\title{
A hybrid zone of the genus Ctenomys: A case study in southern Brazil
}

\author{
Camila S. Castilho ${ }^{1}$, Adriana Gava ${ }^{2}$ and Thales R.O. de Freitas ${ }^{1}$ \\ ${ }^{1}$ Departamento de Genética, Universidade Federal do Rio Grande do Sul, Porto Alegre, RS, Brazil. \\ ${ }^{2}$ Universidade Federal do Rio Grande, Rio Grande, RS, Brazil.
}

\begin{abstract}
We describe variation at microsatellite loci and the chromosomal polymorphisms of a hybrid population, and hybridizing populations of Ctenomys minutus (the minor tuco-tuco) from the coastal plain of Rio Grande do Sul, southern Brazil. Cytogenetic analysis and a survey of six microsatellite loci included 101 specimens of $C$. minutus from the parental populations $(2 \mathrm{n} / \mathrm{AN}=42 / 74$ and $48 \mathrm{a} / 76)$ and their contact zone. Cytogenetic analysis recorded 26 different karyotypes exhibited by 50 individuals from the hybrid population. Of the 26 karyotypes, only $14 \%$ presented a parental-like configuration, and none had the combinations of $2 \mathrm{n}$ and AN expected for an F1 hybrid. The remaining karyotypes were alternative hybrid forms, with 2 n varying from 42 to 46 and AN from 68 to 80 . These results suggest chromosomal rearrangements are only of minor significance in the establishment of reproductive barriers for this species.
\end{abstract}

Keywords: chromosome polymorphism, subterranean rodent, karyotypes, microsatellite.

\section{Introduction}

Hybrid zones occur when genetically distinct groups of individuals meet and mate, resulting in at least some offspring of mixed ancestry (Barton and Hewitt, 1989; Harrison, 1993). They have attracted the attention of evolutionary scientists because the extensive genetic variation that they contain helps to understand evolutionary processes that contribute to their origin and maintenance (Harrison, 1993). Hybrid zones have had a major importance in studies of speciation, especially for evolutionary models, patterns of introgression and species integrity (Hewitt, 1988; Harrison, 1993; Gava and Freitas, 2003, 2004; Trigo et al., 2008). The hybridizing populations may be from the same or different species (Hewitt, 1988; Barton and Hewitt, 1989; Harrison, 1993).

In southern Brazil there is an exceptional opportunity to study hybrid zones in the genus Ctenomys, as there are intra- and inter-specific hybrid zones described here for $C$. minutus (Gava and Freitas, 2002, 2003; Freygang et al., 2004; Gava and Freitas, 2004; Castilho C, 2004, Master's thesis, Universidade Federal de Rio Grande do Sul, Porto Alegre, RS, Brazil). Hybrid zones can also have different forms of variation, for example, a "hybrid swarm", when a diverse array of recombinant types exist, or in other situa-

Send correspondence to Camila S. Castilho. Laboratório de Citogenética e Evolução, Departamento de Genética, Universidade Federal do Rio Grande do Sul, Avenida Bento Gonçalves 9500, Prédio 43323, Caixa Postal 15053, 91501-970 Porto Alegre, RS, Brazil. E-mail: cscastilho@ hotmail.com. tions when only $\mathrm{F}_{1}$ hybrids (in addition to parental types) are found (Harrison 1993). Both of these situations are described along the distribution range of $C$. minutus in southern Brazil (Gava and Freitas, 2002, 2003; Freygang et al., 2004; Gava and Freitas, 2004; Castilho C, 2004, Master's thesis, Universidade Federal de Rio Grande do Sul, Porto Alegre, RS, Brazil).

Hybrid zones that include chromosomal variation, as is the case in several species of Ctenomys, are of major interest because of the continuing controversy regarding the role of chromosomal rearrangements in speciation (Sites and Moritz, 1987; Hewitt, 1988; Barton and Hewitt, 1989). Despite the knowledge that chromosomal changes may cause phenotypic changes, with consequences for the adaptation of organisms to their environment and for changes in interactions between individuals of the same species (summarized by White, 1978), their role as reproductive barriers is still controversial (King, 1987; King, 1993; Rieseberg, 2001; Navarro and Barton, 2003). The idea is that if underdominance of heterozygotes for chromosomal rearrangement is strong, it would be very unlikely that new rearrangements could become established. On the other hand, if underdominance is weak enough for fixation to occur, chromosomal rearrangements would be very poor barriers to gene flow and, thus, unlikely to contribute to speciation (Navarro and Barton, 2003).

Microsatellite markers have been used in studies of hybrid zones, where they have provided insights into introgression, population structure and gene flow (Goodman et al., 1999; Dallas et al., 1998; Gava and Freitas, 2004; Trigo 
et al., 2008) and have also revealed hybrid fertility in backcrosses of hybrids or crosses between hybrids (Harrison, 1993).

The genus Ctenomys comprises approximately 60 living species of rodents and shows impressive intra- and inter-specific karyotypic variation (Reig et al., 1990). C. minutus inhabits sandy fields and dunes on the southern Brazilian coastal plain (Freitas, 1997; Gava and Freitas, 2002; Freygang et al., 2004). It is polytypic, possessing 11 distinct karyotypes: $2 \mathrm{n}=42$, 46a, 46b, 47a, 47b, 48a, 48b, 49a, 49b, 50a, and 50b, and arm numbers (AN) varying from 74 to 78 (Freitas, 1997, 2006; Gava and Freitas, 2002, 2003, 2004; Freygang et al., 2004). The chromosomally divergent populations have a parapatric distribution across the coastal plain, and four intraspecific hybrid zones are known in the contact regions of parapatric karyotypes: i) $46 \mathrm{a} \times 48 \mathrm{a}$; ii) $46 \mathrm{~b} \times 48 \mathrm{~b}$; iii) $42 \times 48 \mathrm{a}$; and iv) $50 \mathrm{~b} \times 48 \mathrm{~b}$ (Gava and Freitas, 2002, 2003, 2004; Freygang et al., 2004 Castilho C, 2004, Master's thesis, Universidade Federal de Rio Grande do Sul, Porto Alegre, RS, Brazil). Two of these intraspecific hybrid zones and an interspecific one (between C. minutus and C. lami) have been recorded and studied with respect to chromosomal dynamics and genetic structures (Gava and Freitas, 2002, 2003).

The survey of Ctenomys hybrid zones included contacts between populations with different degrees of chromosomal divergence (Gava and Freitas, 2003). The cytotype $2 \mathrm{n}=48 \mathrm{a}$ has a wide distribution across $110 \mathrm{~km}$ of the coastal plain. Its northernmost populations interact in a $10-\mathrm{km}$-wide zone of contact with cytotype $2 \mathrm{n}=46 \mathrm{a}$. The cytotypes are differentiated by a single Robertsonian rearrangement, and the contact zone has been studied with regard to details of the origin, evolution and adaptive relationships of the cytotypes (Gava and Freitas, 2002), cranial morphology (Marinho and Freitas, 2000), and genetic differentiation and structure (Gava and Freitas, 2004). In the southernmost part of the distribution of C. minutus, Gava and Freitas (2003) identified a hybrid population in a contact zone between two chromosomal races, $2 n=42$ and $2 n$ $=48 \mathrm{a}$, . Cytotype $2 \mathrm{n}=48 \mathrm{a}$ interacts with populations with $2 n=42$. The latter is a cytotype differentiated by a tandem fusion of two acrocentrics, plus a Robertsonian fusion that created a new, readily distinguishable, large chromosome (Freitas, 1997; Gava and Freitas, 2003). The $2 \mathrm{n}=42$ karyotype is also differentiated from the $2 \mathrm{n}=48$ cytotype by another Robertsonian fusion of 2 acrocentrics. The geographical width of the hybrid zone has not been determined, but the northern $(2 n=48 a)$ and southern $(2 n=42)$ parental-like populations recorded were $14 \mathrm{~km}$ apart (Gava and Freitas, 2003).

Although chromosomal rearrangements are considered to have played a major role in the diversification and speciation of the genus Ctenomys (Reig et al., 1990; Lessa and Cook, 1998; Wlasiuk et al., 2003; Tomasco and Lessa, 2007), this role is still controversial for $C$. minutus.

Considering that chromosomal rearrangements have been of major interest in studies in the genus Ctenomys, and their role being controversial in C. minutus hybrids, we used this contact zone between two parental forms with parapatric distribution and different chromosomal rearrangements as an evolutionary model to study the impact of chromosomal rearrangements as reproductive barriers, and their effect on gene flow between populations with different karyotypes in C. minutus.

\section{Material and Methods}

\section{Samples}

We collected tissue and blood samples from 50 individuals on a gradient consisting of four different localities in the contact region between the chromosomal races $2 \mathrm{n}=$ and $2 \mathrm{n}=48 \mathrm{a}$ (Figure 1; coordinates $30^{\circ} 59^{\prime} \mathrm{S}$ and $50^{\circ} 49^{\prime} \mathrm{W}$ ), in order to determine diploid number and microsatellite variation. A complete map with details on the

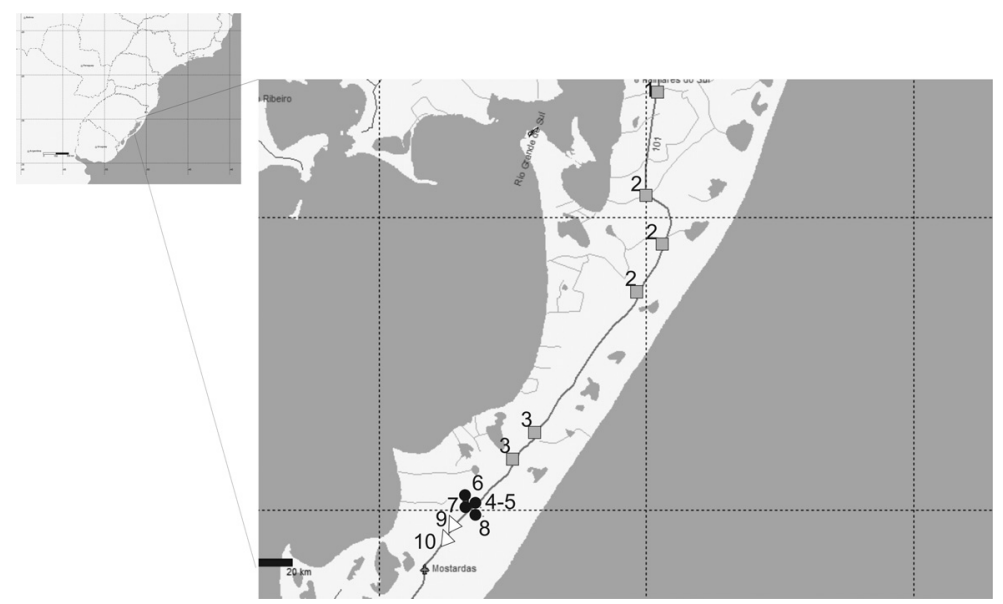

Figure 1 - Sampling sites of individual Ctenomys minutus from populations fixed for $2 \mathrm{n}=48 \mathrm{a}$ (gray squares) or $2 \mathrm{n}=42$ (white triangles), both collected by Gava and Freitas (2003), and polymorphic populations $2 n=42-46$ (black circles), collected for this study from a contact zone on the coastal plain of southern Brazil. Abbreviations for sampling locations are shown in Table 1. 
distribution of Ctenomys minutus, karyotypes, hybrid zones and parental chromosomal rearrangements can be found in Fornel et al. (2010).

Blood samples were collected from hybrid animals using a capillary plus a syringe inserted into the side of the ocular globe of sedated animals. After blood and tissue collection the animals were released at the same spot as they were captured. All tissue samples were deposited in the
Laboratório de Citogenética e Evolução, Departamento de Genética, Universidade Federal do Rio Grande do Sul following the sample number of this collection. The permits for this research were obtained from IBAMA (Permit number 14690-1). For information about sample collection in parental populations see Gava and Freitas (2003).

A total of 51 reference specimens with $2 \mathrm{n}=48 \mathrm{a}(25$ specimens) and $2 \mathrm{n}=42$ (26 specimens; see Freitas, 1997

Table 1 - Sample localities of 101 specimens of Ctenomys minutus. (LN=locality number, $N=$ sample size, $2 n / \mathrm{NA}=$ diploid number and autosomal arm numbers, $P=$ frequency of karyotypes) and Reference= sample collection reference.

\begin{tabular}{|c|c|c|c|c|c|}
\hline Locality & $\mathrm{LN}$ & $N$ & $2 n / \mathrm{NA}$ & $\mathrm{p}$ & Reference \\
\hline Palmares do Sul & 1 & 6 & $48 / 76$ & 1.0 & Gava and Freitas 2003 \\
\hline km 35 Capivari-Tavares Road & 2 & 4 & $48 / 76$ & 1.0 & Gava and Freitas 2003 \\
\hline km 53 Capivari-Tavares Road & 2 & 5 & $48 / 76$ & 1.0 & Gava and Freitas 2003 \\
\hline km 64 Capivari-Tavares Road & 2 & 3 & $48 / 76$ & 1.0 & Gava and Freitas 2003 \\
\hline km 96 Capivari-Tavares Road & 3 & 4 & $48 / 76$ & 1.0 & Gava and Freitas 2003 \\
\hline km 101 Capivari-Tavares Road & 3 & 3 & $48 / 76$ & 1.0 & Gava and Freitas 2003 \\
\hline \multirow[t]{6}{*}{ Capivari-Tavares Road } & $4-5$ & 8 & $44 / 74$ & 0.25 & This study \\
\hline & & & $45 / 76$ & 0.25 & \\
\hline & & & $43 / 70$ & 0.125 & \\
\hline & & & $44 / 73$ & 0.125 & \\
\hline & & & $45 / 74$ & 0.125 & \\
\hline & & & $46 / 76$ & 0.125 & \\
\hline \multirow[t]{5}{*}{ Capivari-Tavares Road } & 6 & 7 & $42 / 74$ & 0.44 & This study \\
\hline & & & $42 / 73$ & 0.14 & \\
\hline & & & $42 / 71$ & 0.14 & \\
\hline & & & $42 / 69$ & 0.14 & \\
\hline & & & $43 / 74$ & 0.14 & \\
\hline \multirow[t]{6}{*}{ Capivari-Tavares Road } & 7 & 6 & $45 / 76$ & 0.17 & This study \\
\hline & & & $42 / 74$ & 0.17 & \\
\hline & & & $45 / 80$ & 0.17 & \\
\hline & & & $45 / 74$ & 0.17 & \\
\hline & & & $46 / 71$ & 0.17 & \\
\hline & & & $46 / 76$ & 0.17 & \\
\hline \multirow[t]{18}{*}{ Capivari-Tavares Road } & 8 & 29 & $42 / 74$ & 0.10 & This study \\
\hline & & & $46 / 74$ & 0.07 & \\
\hline & & & $43 / 75$ & 0.07 & \\
\hline & & & $42 / 72$ & 0.07 & \\
\hline & & & $46 / 77$ & 0.07 & \\
\hline & & & $44 / 76$ & 0.07 & \\
\hline & & & $44 / 75$ & 0.07 & \\
\hline & & & $45 / 78$ & 0.07 & \\
\hline & & & $44 / 74$ & 0.07 & \\
\hline & & & $43 / 73$ & 0.07 & \\
\hline & & & $42 / 68$ & 0.03 & \\
\hline & & & $46 / 78$ & 0.03 & \\
\hline & & & $42 / 71$ & 0.03 & \\
\hline & & & $43 / 72$ & 0.03 & \\
\hline & & & $44 / 72$ & 0.03 & \\
\hline & & & $45 / 76$ & 0.03 & \\
\hline & & & $42 / 70$ & 0.03 & \\
\hline & & & $43 / 74$ & 0.03 & \\
\hline km 115 Capivari-Tavares Road & 9 & 3 & $42 / 74$ & 1.0 & Gava and Freitas 2003 \\
\hline km 120 Capivari-Tavares Road & 10 & 23 & $42 / 74$ & 1.0 & Gava and Freitas 2003 \\
\hline
\end{tabular}


for more details on parental karyotypes). In this work we used the same set of microsatellite loci as previously employed by Gava and Freitas (2003) (Table 1).

\section{Cytogenetic methods}

The blood was seeded in AIM-V culture medium (Gibco) and incubated at $37^{\circ} \mathrm{C}$ for $70 \mathrm{~h} .50 \mathrm{~mL}$ of colchicine $(0.025 \%)$ was then added and the solution incubated for $2 \mathrm{~h}$ at $37^{\circ} \mathrm{C}$. The cultures were treated with hypotonic solution $(0.075 \mathrm{M} \mathrm{KCl})$ for $15 \mathrm{~min}$ and then fixed with methanol:acetic acid (3:1). Slides were prepared and conventionally stained with Giemsa for determining diploid numbers.

\section{DNA extraction and microsatellite loci analysis}

DNA from individuals of mixed ancestry was isolated from the tissue samples using the method described by Medrano et al. (1990) with modifications. Six polymorphic microsatellites isolated from C. haigi, with dinocleotide (Hai2; Hai3; Hai4; Hai5; and Hai6) and trinucleotide repeats (Hai12) were employed (Lacey et al., 1999). Amplification reactions were performed in volumes of $20 \mu \mathrm{L}$ (50 ng of genomic DNA, $0.4 \mu \mathrm{M}$ of each primer, $0.4 \mathrm{mM}$ dNTP, 1x Taq Buffer, $1.5 \mathrm{mM} \mathrm{MgCl}_{2}$ and $0.8 \mathrm{U}$ Taq DNA Polymerase). The conditions used were: denaturation at $94{ }^{\circ} \mathrm{C}$ for $5 \mathrm{~min}, 30$ cycles with annealing temperatures from $52-60{ }^{\circ} \mathrm{C}$ for the different primer sets, ending with a final extension of $5 \mathrm{~min}$ at $72{ }^{\circ} \mathrm{C}$. PCR products were separated in $8 \%$ denatured polyacrylamide gels and stained with silver nitrate. A 25 bp DNA ladder was used to score the genotypes, and individuals were replicated in different gels to certify the allele lengths.

\section{Statistical analysis}

The number of polymorphic loci, number of alleles per locus and number of exclusive alleles per population determined by Arlequin 3.1 (Schneider et al., 2000) were used to estimate genetic variability. Observed heterozygosity (Ho), expected heterozygosity (HE) and HardyWeinberg equilibrium were estimated for all loci and all populations using the Markov Chain Method (Guo and Thompson, 1992) implemented in Genepop 3.4 (Raymond and Rousset, 1995) and Arlequin 2.1. Linkage disequilibrium was tested between loci in all populations using Genepop 3.1. These results were adjusted with a Bonferroni correction (Rice, 1989). Values of $F_{I S}$ were calculated using the method of Weir and Cockerham (1984) as in Genepop. The probability of the presence of null alleles, allele dropout, and scoring errors due to stutter was tested in global data set using MicroChecker 2.2.3 (Van Oosterhout et al., 2004). A locus-by-locus Analysis of Molecular Variance (AMOVA) (Excoffier et al., 1992) was performed to infer population genetic structure through the fixation index $F_{S T}$ implemented in Arlequin 2.1.
To assess the existence of population structure, we used a Bayesian model-based clustering method implemented in STRUCTURE 2.3.3 (Pritchard et al., 2000), using all 101 samples and six microsatellite loci. We conducted five independent runs for each number of clusters (k) between 1 and 15, using the admixture model, no prior population information, 500,000 burn-in and $10^{6}$ replications. We also used a principal components analysis (PCA) of the autosomal gene-frequency data to investigate spatial patterns of genetic variation, using PCAGEN.

\section{Results}

\section{Chromosomal polymorphism}

We recorded variation in $2 \mathrm{n}$ from 42 to 46 and AN from 68 to 80, distributed across 26 different karyotypes of 50 individuals from the contact zone (Table 1). None of the karyotypes showed $2 \mathrm{n}=48 \mathrm{a}$ and $\mathrm{AN}=76$, typical for the northern sampling sites or the $\mathrm{F} 1$ configuration $2 \mathrm{n}=45$ and $\mathrm{AN}=75$, expected in crosses between parental types. The $2 n=42$ and $A N=74$ configuration was found in $14 \%$ of the specimens. Homokaryotypes comprised $46 \%$ of the sample, whereas $54 \%$ of it was composed of heterozygotes for one ( $28 \%$ fusion/fission) or another rearrangement $(20 \%$ of pericentric inversions) or both rearrangements $(6 \%$ fusion/fission and pericentric inversions).

\section{Microsatellite polymorphism, heterozygosity and linkage disequilibrium}

The six loci analyzed were highly polymorphic. The number of alleles scored was 67 for all loci in the three populations (both parental and hybrid), and 36 (54\%) of them were exclusive to one of the populations. Forty-eight alleles were found in the reference $2 n=48$ a population, of which 18 were not found in the hybrid or $2 n=42$ population. Twelve of 43 alleles found for the reference $2 n=42$ population were not found in either the $2 \mathrm{n}=48 \mathrm{a}$ or the hybrid population. Forty-eight alleles were found in the hybrid population, of which six were not found in either of the parental populations (Table 2). The most variable locus was Hai6, with 19 alleles (allele size ranges: 120-154 bp). The least variable loci were Hai2 (169-181 bp) and Hai5 (201$211 \mathrm{bp}$ ) with 7 and 6 alleles, respectively. Hai4; Hai3 and Hai12 showed 15 (158-160/164-184/188-190 bp), 11 (150-170/174 bp), and 9 alleles (120-144 bp) respectively. Analysis of linkage disequilibrium, after Bonferroni correction, suggested lack of genetic linkage among pairs of loci across all populations.

The contact population showed values of heterozygosity that deviated from Hardy-Weinberg equilibrium ( $p<0.05$; after Bonferroni correction), with a heterozygote deficit in five of six loci analyzed (except for Hai5). A heterozygote deficit was also detected in most loci of the parental populations, except for Hai 5 and Hai1 2 for population $2 \mathrm{n}=48 \mathrm{a}$, and Hai3, Hai4, Hai5, and Hai12 for popula- 


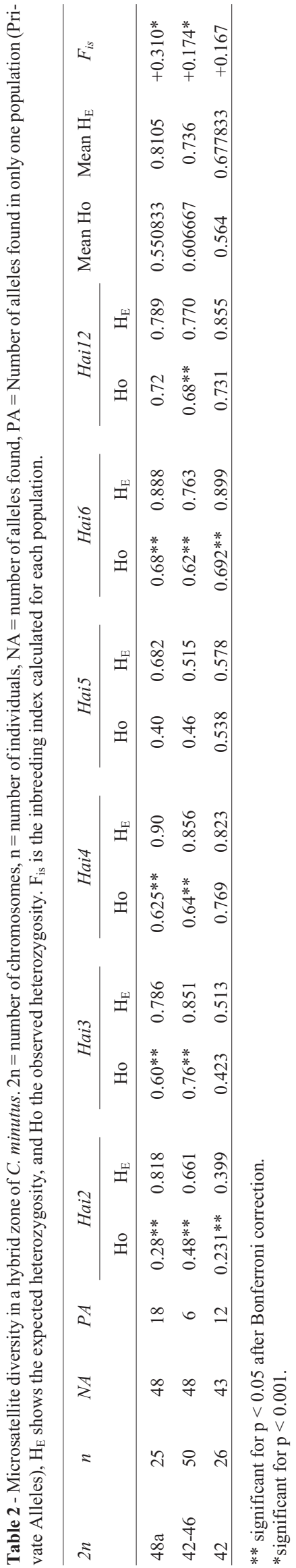

tion $2 n=42$ (Table 2). MicroChecker results gave no evidence of allele dropout, although three loci (Hai 2, Hai 4 and Hai 6) presented a general excess of homozygotes for most allele-size classes, thereby implying the possible presence of null alleles. The $F_{I S}$ value calculated for all populations was positive and highly significant (Table 2). Allelic frequencies of 11 alleles from different loci (175 bp-Hai2; 150, 152, 158 and 160 bp-Hai3; 164 bp-Hai4; 203 and 209 bp-Hai5; 134 and 152 bp-Hai6; 120 bp-Hai12) showed clinal variation, with the contact zone showing intermediate frequencies to those of the parental populations.

$F_{\mathrm{ST}}$

The genetic structure of the populations was investigated by the fixation index $F_{\mathrm{ST}}$. The fixation index supported a greater genetic divergence among the parental population $2 \mathrm{n}=42$ and $2 \mathrm{n}=42-46\left(F_{\mathrm{ST}}=0.13877\right.$; $\mathrm{p}<0.001$ ) than among populations $2 \mathrm{n}=48 \mathrm{a}$ and $2 \mathrm{n}=42-46$ $\left(F_{\mathrm{ST}}=0.10040 ; \mathrm{p}<0.001\right)$.

\section{Population structure}

Bayesian clustering analyses through the software STRUCTURE indicated that there are eight genetic populations in our sample (Figure 2). We could observe that hybrid individuals share more genetic similarity to parental $2 \mathrm{n}$ $=48 \mathrm{a}$, especially in sample spots near to the hybrid zone and that parental populations share very little genetic similarities between them. Nonetheless, we found that in hybrid sample site 8 one individual was genetically similar to the parental $2 \mathrm{n}=42$ genetic populations.

\section{Principal components analysis (PCA)}

Patterns of genetic differentiation were represented by a multivariate PCA plot (Figure 3 ). The individual PCA scores, plotted on the first two principal components axes, PC-I and PC-II, explained $13.54 \%$ and $9.54 \%$ respectively of the total genetic diversity. Samples from parental $2 n=42$ clustered in the upper left part of the PCA plot, whereas samples from parental $2 n=48$ a were in the lower part. Hybrid samples clustered in the upper part and lower right part, and grouped with $2 n=42$ in the upper left and with $2 n$ $=48 \mathrm{a}$ in the lower right quadrant (Figure 2).

\section{Discussion}

Among the 50 specimens analyzed from the hybrid zone between the parental karyotypes, we found a high percentage of hybrid forms ( $86 \%$ ). Considering the variation in $\mathrm{AN}$ from 68 to 80 we infer that these variations cannot be produced only by hybrid and parental-like genotypes or parental and parental crosses. Accordingly, in this zone, hybrids would have been able to breed with other hybrids and there is no evidence of hybrid infertility in this hybrid zone in C. minutus. This finding is also the first recording of AN less then 74 and higher then 78 for C. minutus. 


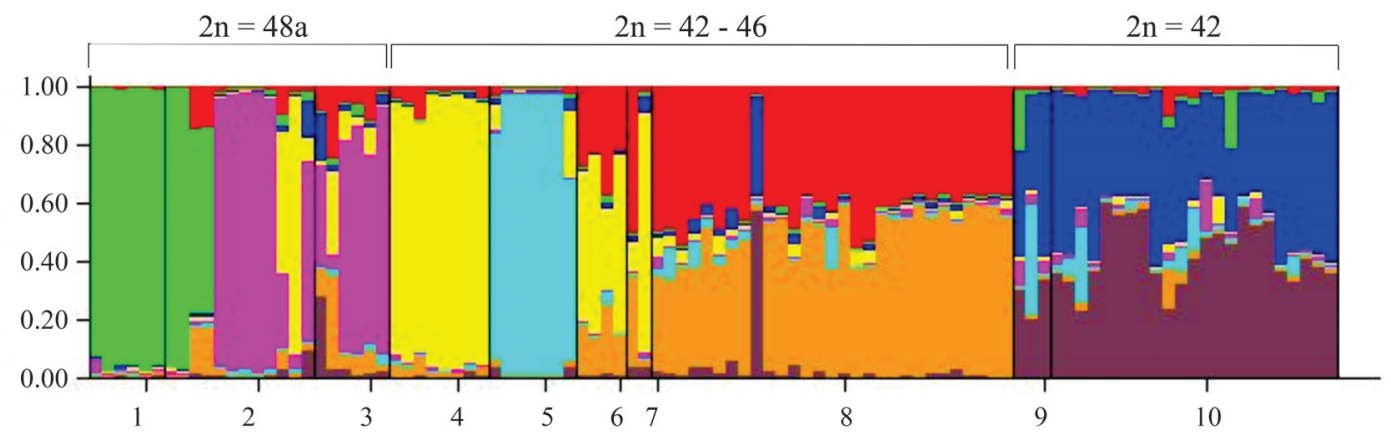

Figure 2 - Structure bars plot showing the eight genetic populations identified by the analysis. The numbers 1-10 correspond to the sample spots in Figure 1 and Table 1.

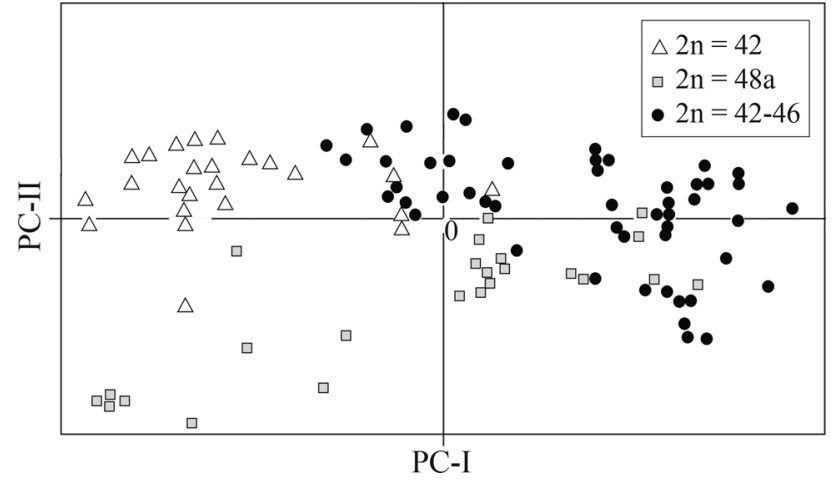

Figure 3 - Scores of individual microsatellite genotypes of $C$. minutus plotted on the first two axes (PC-I, PC-II) of a principal components analysis.

In addition to the chromosomal evidence, the PCA analysis showed overlap between the parental and hybrid populations, as also corroborated by the structure analysis which showed that parental and hybrid populations share genetic similarities. We registered alleles that are shared among populations and 11 alleles on six loci that exhibited a clinal distribution along the zone, indicating gene flow across the hybrid zone and parental individuals. The $F_{S T}$ results indicated that these crosses appear to occur more frequent between hybrids and parental $2 \mathrm{n}=48 \mathrm{a}$ than within parental $2 \mathrm{n}=42$. The PCAGEN and Structure results pointed into the same direction.

Gava and Freitas (2003) proposed that the chromosomal variation described for $C$. minutus does not reduce hybrid fitness and acts as a transitory polymorphism in populations. Their conclusion that the role of chromosomal variability for this species as a reproductive barrier and speciation factor is still controversial is also in line with our current data.

The studies of populations and loci showed a significant heterozygote deficit for most loci in all populations, with a highly significant and positive $F_{I S}$, and significant deviations from Hardy-Weinberg equilibrium. For $C$. minutus, a heterozygote deficit has also been reported in another intraspecific hybrid zone (Gava and Freitas, 2004). In a study done in Europe on hybridization between wolves and domestic dogs using microsatellites, Randi and Lucchini (2002) found significant deviations from Hardy-Weinberg equilibrium, a heterozygote deficit, and positive

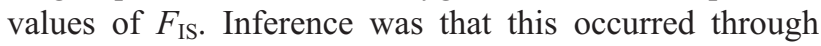
non-random breeding due to inbreeding or geographically structured subpopulations. This may also explain the deficit observed in our study as the species studied live in populations that are structured even on a small geographic scale and dispersed over short distances only. These characteristics may cause population isolation or preferential mating, both resulting in endogamy. Although, these deviations are also to be expected when the analyses are performed in different pooled populations and are explained, most probably, by the Wahlund effect. This would have been the result of an amalgamation of populations with divergence in allelic frequencies, and evidence for this is apparent in the Structure bar plot (Figure 2) where samples sites 1 and 2 are genetically very different from other samples sites. Finally we observed that three of the six loci may have null alleles, this possibly contributing to the deviation from the expected.

Throughout the hybridization zone we found in this study an intermediate distribution of 11 alleles at six loci and a cytotype of $2 n=42 / A N=74$, in addition to the $54 \%$ exclusive alleles found in only one or another population. This may indicate a secondary origin for this zone. In an earlier study on a hybrid zone for C. minutus using microsatellite loci, Gava and Freitas (2004) found that 39.2\% of alleles were present in only one or the other population, as well as the existence of coincident clines in alleles and chromosomes throughout the zone. They concluded that the zone between individuals $2 \mathrm{n}=48 \mathrm{a}$ and $46 \mathrm{a}$ is secondary in origin.

We can also classify this zone as unimodal (Harrison and Bogdanowicz, 1997) because of the predominance of intermediate hybrid genotypes. Across this hybridation zone no environmental variation was observed between areas occupied by the parent stocks and the hybrids, except for distance and an environment with a patchy distribution 
of areas suitable for Ctenomys occupation (Gava and Freitas, 2004).

In conclusion, as we observed several intermediate hybrid forms and evidence of gene flow between parental and hybrid individuals, we suggest that chromosomal rearrangements have minor significance on the establishment of reproductive barriers for Ctenomys minutus.

\section{Acknowledgments}

We would like to thank S.L. Althoff and J.R. Marinho for their important help in fieldwork, and Charlie Baer for suggestions on early versions of the manuscript. This research was financially supported by the Conselho Nacional de Desenvolvimento Científico e Tecnológico (CNPq) and the Fundação de Amparo à Pesquisa do Rio Grande do Sul (FAPERGS).

\section{References}

Barton NH and Hewitt GM (1989) Adaptation, speciation and hybrid zones. Nature 341:497-503.

Dallas JF, Bonhomme F, Boursot P, Britton-Davidian J and Bauchaus V (1998) Population genetic structure in a Robertsonian race of house mice: Evidence from microsatellite polymorphism. Heredity 80:70-77.

Excoffier L, Smouse P and Quattro J (1992) Analysis of molecular variance inferred from metric distances among DNA haplotypes: Applications to human mitochondrial DNA restriction data. Genetics 131:479-491.

Fornel R, Cordeiro-Estrela P and Freitas TRO (2010) Skull shape and size variation in Ctenomys minutus (Rodentia, Ctenomyidae) in geographical, chromosomal polymorphism, and environmental contexts Biol J Linn Soc 101:705-720.

Freitas TRO (1997) Chromosome polymorphism in Ctenomys minutus (Rodentia-Octodontidae). Rev Bras Genet 20:1-7.

Freitas TRO (2006) Cytogenetics status of four Ctenomys species in the south of Brazil. Genetica 126:227-235.

Freygang CC, Marinho JR and Freitas TRO (2004) New karyotypes and some considerations about the chromosomal diversification of Ctenomys minutus (Rodentia, Ctenomyidae) on the Coastal Plain of the Brazilian state of Rio Grande do Sul. Genetica 121:125-132.

Gava A and Freitas TRO (2002) Characterization of a hybrid zone between chromosomally divergent populations of Ctenomys minutus (Rodentia, Ctenomyidae). J Mammal 83:843-851.

Gava A and Freitas TRO (2003) Inter and intra-specific hybridization in tuco-tucos (Ctenomys) from Brazilian Coastal Plains (Rodentia, Ctenomyidae). Genetica 119:11-17.

Gava A and Freitas TRO (2004) Microsatellite analysis of a hybrid zone between chromosomally divergent populations of Ctenomys minutus from Southeastern Brazil (Rodentia, Ctenomyidae). J Mammal 85:1201-1206.

Goodman SJ, Barton NH, Swanson G, Abernethy K and Pemberton JM (1999) Introgression through rare hybridization: A genetic study of a hybrid zone between red and sika deer (genus Cervus) in Argyll, Scotland. Genetics 152:355-371.

Guo S and Thompson EA (1992) Performing the exact test of Hardy-Weinberg proportion for multiple alleles. Biometrics 48:361-372.
Harrison RG (1993) Hybrids and hybrid zones: Historical perspective. In: Harrison RG (ed) Hybrid Zones and the Evolutionary Process. Oxford University Press, Oxford, pp 3-12.

Harrison RG and Bogdanowicz SM (1997) Patterns of variation and linkage desequilibrium in a field cricket hybrid zone. Evolution 51:493-505.

Hewitt GM (1988) Hybrid zones natural laboratories for evolutionary studies. Trends Ecol Evol 3:158-167.

King M (1987) Chromosomal rearrangements, speciation and the theoretical approach. Heredity 59:1-6.

King M (1993) Species Evolution. Cambridge University Press, Cambridge, $335 \mathrm{pp}$.

Lacey EA, Maldonado JE, Clabaugh JP and Matocq MD (1999) Interspecific variation in microsatellites isolated from tucotucos (Rodentia, Ctenomyidae). Ecology 8:1754-1756.

Lessa EP and Cook JA (1998) The molecular phylogenetics of tuco-tucos (genus Ctenomys, Rodentia, Octodontidae) suggests an early burst of speciation. Mol Phylogenet Evol 9:88-99.

Marinho JR and Freitas TRO (2000) Intraspecific craniometric variation in a chromosome hybrid zone of Ctenomys minutus (Rodentia, Hystricognathi). Int J Mam Biol 65:226-231.

Medrano JF, Aasen AND and Sharrow L (1990) DNA Extraction from nucleated red blood cells. Biotechniques 8:43.

Navarro A and Barton NH (2003) Chromosomal speciation and molecular divergence-accelerated evolution in rearranged chromosomes. Science 300:321-324.

Pritchard JK, Stephens M and Donelly P (2000) Inference of population structure using multilocus genotype data. Genetics 155:945-959.

Randy AND and Lucchini V (2002) Detecting rare introgression of domestic dog genes into wild wolf (Canis lupus) populations by Bayesian admixture analyses of microsatellite variation. Conserv Genet 3:31-45.

Raymond M and Rousset F (1995) Genepop ver. 1.2: Population genetics software for exact test ecumenism. J Hered $86: 248-249$

Reig OA, Busch C, Ortellis MO and Contreras JR (1990) An overview of evolution, systematics, population and speciation in Ctenomys. In: Nevo AND and Reig OA (eds) Evolution of Subterranean Mammals at the Organismal and Molecular Levels. A.R. Liss, New York, pp 71-96.

Rice WR (1989) Analyzing tables of statistical tests. Evolution 43:223-225.

Rieseberg L (2001) Chromosomal rearrangements and speciation. Trends Ecol Evol 16:351-358.

Schneider S, Kueffer JM, Roesli D and Excofier L (2000) Arlequin ver. 2.1: Software for population genetic data analysis. Genetic and Biometry Laboratory, University of Geneva, Geneva, Switzerland.

Sites JW and Moritz C (1987) Chromosomal evolution and speciation revisited. Syst Zool 36:53-174.

Tomasco I and Lessa EP (2007) Phylogeography of the tuco-tuco Ctenomys pearsoni: mtDNA variation and its implication for chromosomal differentiation. In: Kelt DA, Lessa EP, Salazar-Bravo JA and Patton JL (eds) The Quintessential Naturalist: Honoring the Life and Legacy of Oliver P. Pearson. University of California Publication in Zoology Series, Berkeley, pp 859-882. 
Trigo TC, Freitas TRO, Kunzler G, Cardoso L, Silva JCR, Johnson WE, O'Brien SJ, Bonatto SL and Eizirik E (2008) Inter-species hybridization among Neotropical cats of the genus Leopardus, and evidence for an introgressive hybrid zone between L. geoffroyi and L. tigrinus in southern Brazil. Mol Ecol 17:4317-4333.

Van Oosterhout C, Hutchinson WF, Wills DPM and Shipley P (2004) MICRO-CHECKER: Software for identifying and correcting genotyping errors in microsatellite data. Mol Ecol Notes 4:535-538.

Weir BS and Cockerham CC (1984) Estimating F-statistics for the analysis of population structure. Evolution 38:1358-1370.

White MJD (1978) Modes of Speciation. WH Freeman, San Francisco, $455 \mathrm{pp}$.
Wlasiuk G, Garza JC and Lessa EP (2003) Genetic and geographic differentiation in the Rio Negro tuco-tuco (Ctenomys rionegrensis) inferring the roles of migration and drift from multiple genetic markers. Evolution 57:913-926.

\section{Internet Resources}

PCAGEN software, http://www2.unil.ch/popgen/softwares/pcagen.htm (July 15, 2003).

License information: This is an open-access article distributed under the terms of the Creative Commons Attribution License, which permits unrestricted use, distribution, and reproduction in any medium, provided the original work is properly cited. 